\title{
The Bone Renaissance- From Conception to Present
}

\author{
Mahmood Hussain Qureshi* \\ Qureshi Dental Clinic, Pakistan
}

Submission: February 28, 2017; Published: March 27, 2017

*Corresponding author: Mahmood Hussain Qureshi, President, Pakistan Academy of Implant Dentistry, Director, MQI- the International Institute of Implant \& Reconstructive Surgery, The Qureshi Dental Centres: MQ Implant Institute, 239-D2, Johar Town, Lahore, 1051-B, LMQ Road, Multan-60700, Pakistan, Tel: +92 300 4134819; Email: mqimplants@gmail.com

\begin{abstract}
In a relentless pursuance of perfection and a definitive solution for long term stability of tissues around implants, the author will present an exceptional concept, 'The Bone Renaissance', a unique philosophy encompassing the sequential and codified reversal of the bone back to its original 3-D Engineered Divine Osseo-architecture (com'era, dov'era); by incorporating its components: transmucosal fixtures with autologous growth factors and cervix perimeters analogous to replaced teeth with stable alveolar bone, soft tissue management, vascularized osteotomies, onlay \& sinus grafts. The ensuing resurrection of the lost contours of the hard and soft tissues achieved primarily by treating the bone and soft tissue deficiencies encompasses a long-term success and esthetic predictability by virtue of stable alveolar bone implant reconstructive integration naturally; 5 in 1 modus operandi- a distinctive enterprise of treating the untreatable patients- a major paradigm shift in re-establishing the natural spiritual union of the form and function.
\end{abstract}

Keywords: Endosseous implants, Flapless, Osteotomes, Soft hard tissue manipulation, Sinus grafts, Expansion, Autologous growth factors Bone renaissance

\section{Introduction}

Implantology has always been overflowing with many diverse thoughts, fads, or commercially promoted products and concepts. Over the past half a century or so, more than $80 \%$ of products and subjects, which were highly promoted for a period of time, proved to be the clinical failures and are redundant now .Regrettably today's implant practice is mostly commercial - guided and often flawed, erroneous, and inaccurate information is portrayed as the highest level. Life, at times, tends to go "full-circle," and what was accepted falls out of favor only to return with renewed vigor.

In the early days of contemporary implantology, 'immediate load' was practiced only to fall out of favor; in the 1970s, rootform implants with 2-stage protocol became the more common implant modality; yet, the past 15 years has seen a resurgence of immediate load and immediate placement which today are acknowledged.

Contemporary is the architectural era of dental implantology which has drastically changed in the last decade or so. Implantology has become very restorative-driven today and per se the location of the restoration dictates where the implant needs to be placed and as such sets the draft for the surgical aspect of treatment. Prosthodontic compromises due to lack of bone are no longer accepted and the approach "that's where the bone was" - which was so rampant and led to the prosthodontic compromises is no longer the accepted standard. Implantology begins after the Implants have healed and keeping them animate in the patient for a life span is the ordeal. The seal is the deal; perfect seals and the implant may well last a lifetime.

There is a reverence for what God originally granted, and treatment today is directed at restoring that form and function. The phrase 'form follows function' has been misunderstood; form and function should be one, joined in a spiritual union by that I mean soft \& hard tissue architecture. Our goal as implantologist should be to overcome the bone deficiencies and restore the lost tissues. Mature edentulous sites have lost bone from the facial (horizontally deficient, thin), vertical aspects and in the maxillary posterior areas due to sinus enlargement owing to a variety of reasons: tooth loss, disease, trauma, periodontal lesions, cervical caries, removable dentures and systemic diseases [1].

The loss of teeth stops the bone deformation from tooth function and results in the reduction of the inorganic component of bone, durahydroxylapatite [2]. Anatomic consequences of tooth loss are: loss of bone, soft tissue changes, loss of contours, devastating aesthetic results, decreased vertical dimensions, deepening lines, wrinkles; thinning of lips, class 3, ptosis of mentalis muscle attachment (witch's chin). 
The single most upsetting factor faced by a surgeon who desires to reproduce a natural contoured fixed prosthesis is bone deficiency which is an inevitable sequel to the loss of teeth. To place each implant in the same 3-dimensional position as the root it replaces is the real challenge to persistently accomplish. This was the challenge we recognized in the beginning of career, back in early nineties and even today, only a small part of our professionals recognizes this as a primary target, with even smaller fraction capable to clinically produce these domino effects. These objectives should be accomplished with the simplest, most cost-effective methods. Our object of implant treatment has always been the restoration and lifelong maintenance of patients to innate aesthetics and function. The real test is how we can make all of this take place regularly, predictably and economically. We need a paradigm swing in our contemplation on how we recreate bone.

The Bone Renaissance is the culmination of over 25 years in the search for knowledge, comprehension and experience in the field of Implantology, and as a matter of fact, roots of research and invention actually goes further back at the time of conception of this novel technique by Dr Hilt Tatum in 1970 who coined the term bone manipulation with the specially designed osteotomes.

It is imperative to understand that the bone is visco-elastic, plastic, pliable, flexible, malleable and can be compressed and manipulated. Bone compaction [3] with osteotomes creates a denser bony interface with increased bone to implant contact and therefore good initial stabilization of the dental implant. Osteotomes can offer several significant advantages over the traditional graded series of drills. Osteotomes take advantage of the fact that bone is visco-elastic and can be compressed and manipulated. Compression creates a denser area for implant placement. This technique also allows for greater tactile sensitivity with the three dealings: compaction, expansion, cortical floor elevation: sinus and nasal lift- a procedure where the cortical plate and lining of the sinus floor is tapped and moved up [4] to gain an additional height of bone about $3-5 \mathrm{~mm}$ and these all can be combined to facilitate implantation. If the practitioner recognizes the properties of bone and understands how bone responds to manipulation, the techniques described here can aid in the preparation for the placement of dental implants with greater

success (Figure 1).

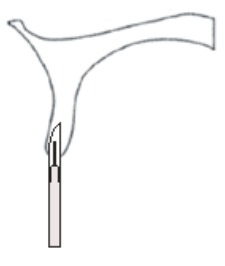

a
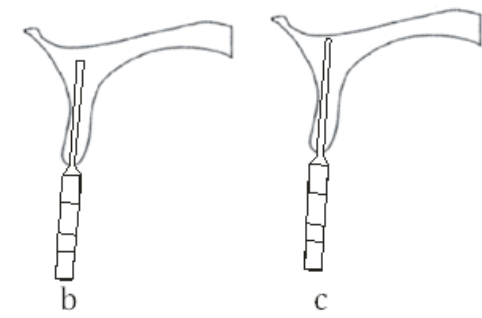

c

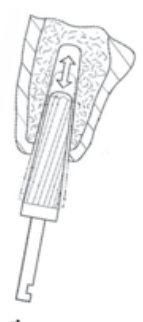

d

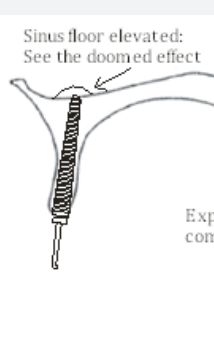

$\mathrm{e}$

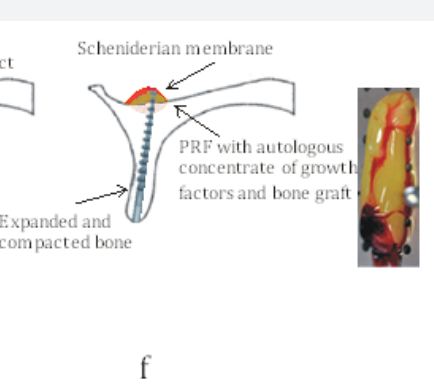

f

Figure 1: Technique to expand the narrow ridge with simultaneous sinus lift in conjunction with implant placement \& concentrated autologous growth factors.

The above illustration shows a narrow $2 \mathrm{~mm}$ wide and $5 \mathrm{~mm}$ long maxillary ridge; osteotomy initiated with blade \#15 to separate the buccal and the palatal plates; pointed $2 \mathrm{~mm}$ osteotome tapped in up to $4 \mathrm{~mm}$ depth followed by a series of rotary and manual osteotomes, progressing in diameter, to expand up to $3.5 \mathrm{~mm}$; blunt osteotome of $4.0 \mathrm{~mm}$ is taken and the bone around the osteotomy is shaved, chiseled and tapped up in the prepared hole along with the autogenous bone against the sides of the prepared socket and PRF membrane inserted in the fractured sinus floor. In due course the cortical plate and lining of sinus floor is lifted simultaneously thereby lifting it up by $3-4 \mathrm{~mm}$ to tent the bone using the elasticity of the scheniderian membrane to give a domed effect; next a $4.5 \mathrm{~mm}$ blunt instrument is engaged to perfect the prepared socket followed by implant placement.

The general entrenched dogma is that the ridges less than $5 \mathrm{~mm}$ requires some form of augmentation procedure in order to receive a fixture. The author measured 219 edentulous ridges in the anterior pre-maxillary area to find out that more than $75 \%$ of ridges in the central incisor and canine area were less than $3.5 \mathrm{~mm}$ wide, whereas the width in the lateral areas was even lesser than $3 \mathrm{~mm}$. Now if the above mentioned patients were to have fixtures inserted, then, according to the routine trends, they were supposed to undergo multiple surgeries and a long waiting period of not less than 12 months or even more, before they were finally restored; which is not acceptable to most of the patients that we see in our practices.

The author has successfully treated more than 11000 ridges both in the maxilla \& mandible, 1-2-3mm wide and expanded them to desirable width; at times alone and in some cases amalgamating and burnishing particulate grafts in conjunction with simultaneous expansion, molding and manipulation with a success rate of well over 95\%. Expansion of even $1 \mathrm{~mm}$ ridges is possible with experience and proficiency, in both upper and lower jaws. Januário et al. studied 250 CBCT scans, reported the mean buccal plate thicknesses in the maxillary anterior region varying from 0.5 to $0.7 \mathrm{~mm}$ (Figure 2) [5]. 


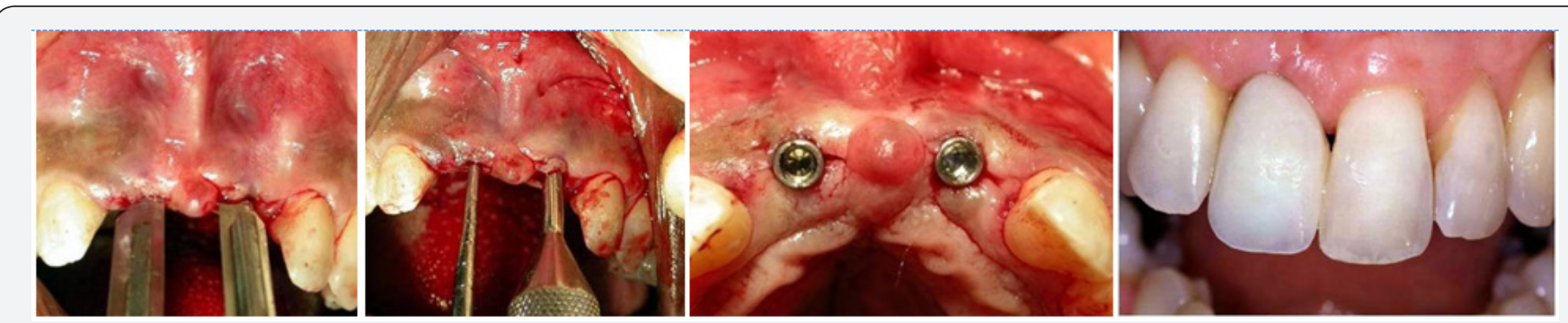

Figure 2: $1 \mathrm{~mm}$ ridge expanded by bone renaissance to receive a $4.5 \mathrm{~mm}$ implant followed by ideal restorations.

In very thin ridges screw implants are not appropriate; specially designed implants with a reduced diameter of almost $50 \%$, having finns to increase the surface area for stress distribution. They are so designed to fit into the expanded ridge and contribute to the formation of an increased volume of stable, supporting bone.

All endosteal fixtures more or less can be placed with flapless surgery to curtail vascular insults towards the alveolar bone in order to preserve its blood supply and reduce crestal bone resorption at the time of implant placement. Bone manipulation can shape the socket in the accurate location even if thin vertical bone is present as long as it remains attached with the overlying periosteum and attached tissues and can be expanded to regain width (Figure 3).

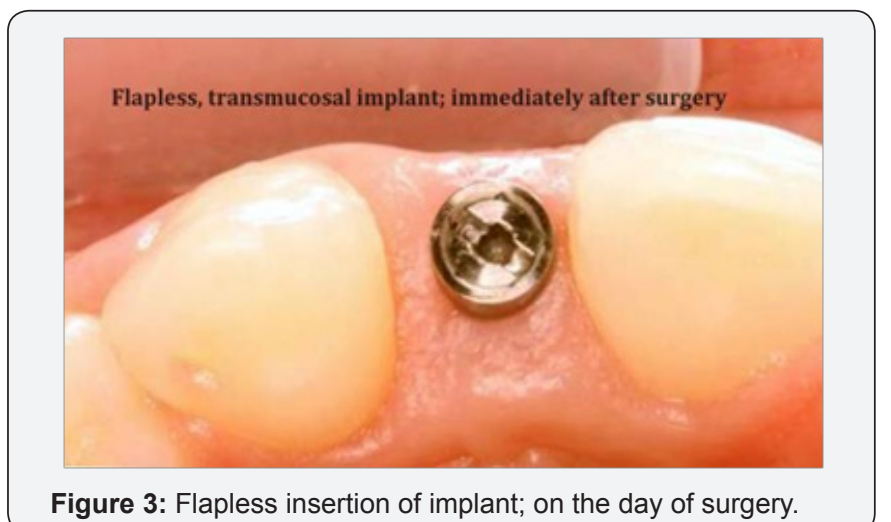

Figure 3: Flapless insertion of implant; on the day of surgery.

Ridge opening, separating, expanding, corticalizing, compacting and manipulating are highly technique sensitive but at the end, swathe enormous unparalleled advantages over the other conventional methods.

Bone Renaissance is a comprehensive solution in the search for success and stability where, by incorporating a complete range of implant modalities, a variety of implant designs; screws, cylinders, finns, blades, ramus frames etc we bring back bone and soft tissue form in its original location, dimension \& quality. It is not restricted to drilling holes and screwing in implants and is not limited or dictated by the commercial implant companies but helps accomplish our challenge of "the reconstruction and preservation of hard and soft tissues to innate aesthetics and function, predictably, routinely, effectively and economically and to place new teeth and bone back to its original 3-D engineered Divine osseo-architecture.

Today-over $90 \%$ of all root-form implants we place are done either completely or partially with bone renaissance concept.

\section{Bone Renaissance Treating the Horizontal Deficiencies}

The Bone Renaissance concept allows the facility of easy access to unapproachable or difficult access areas/complex sites - thus provides universal intraoral use, an increased control of the bone expansion, which facilitates implant-site preparation.

Multiple procedures (5 surgeries) are avoided and the patient is treated with a single surgical procedure. It enhances the clinician's ability to manipulate ideal socket development in Type I, II, III, and IV bone, while significantly reducing the potential complications caused by the percussive trauma generated with the osteotome technique alone.

The codified combination of smooth, blunt, tapered, pointed, cylindrical, blunt \& threaded design of the manual \& motordriven bone expanders helps in desirable socket development that facilitates the subsequent insertion of an ideal implant, and promotes initial stability as well.

It also enhances the ability to manipulate and expand the alveolar walls and inter-radicular crests, thereby providing an increased number of sites that may be amenable to immediate postextraction implant placement and avoid the need for multiple-stage procedures. Enlarging steps comprises of simultaneous rotation and pushing the working end of the tapered osteotome into the osteotomy so that one or more burnishing edges concentrate the pushing and rotational force from end of the burnishing edge in outward, normal and tangential component of forces against the interior surface of the osteotomy to incrementally expand the osteotomy with little to no removal of bone material; inserting the tapered working end of the osteotome into an initial osteotomy, enlarging it by forcibly advancing the osteotome into the initial osteotomy.

Bone Renaissance concept uses transmucosal fixtures with autologous growth factors and cervix perimeters analogous to 
replaced teeth with stable alveolar bone to establish a natural harmony between diameters of implants \& crowns as a result of having a choice of implant sizes which will correlate to the dimensions of the clinical crowns to be utilized. Implant diameters of $2.5,3.0,3.2,3.5,4.0,4.5,5.0,6,7,8 \mathrm{~mm}, 10 \mathrm{~mm}$ \& Lengths from 6 $\mathrm{mm}$ to $25 \mathrm{~mm}$, are used with a variety of implant designs \& shapes. This choice, combined with the ability to create bone sockets in the correct position with Bone Renaissance, allows this desired relationship of harmony to be predictability created.

Bone Renaissance is an atraumatic, innovative, flapless surgical technique developed for expanding an initial osteotomy to receive an endosseous implant by incorporating a combination of mallet-driven \& motor-driven bone expanders together with an amalgamation of autologous growth factors to be used in a sequential, codified approach. Bone renaissance allows us to treat horizontally thin \& vertically deficient ridges. In almost all situations in both upper and lower arches where vertical height is present, bone manipulation can create normal sockets, as long as periosteum and blood supply are intact [6], bone is plastic and will heal into its new shape. Implants are best placed with flapless surgery to protect vascularity and with minimal bone removal to best utilize the reduced bone mass. When the bone is expanded, overlying periosteum is also stretched and as of this blood supply from periosteum tension is released in cortical bone. Periosteum also aids bone support and helps in rebuilding the fractured bone segments caused by tapping and expanding.

Implant Companies tend to promote their own products; strict protocols dictated by implant companies, promote their own systems, reduce the choice of the most suitable treatment for the patients. Drilling and cutting take bone away from the implant site; heat is a major detriment to osseointegration produced with the use of drills; moreover during the drilling process, there is no practical means to immediately improve adjacent bone quality. Correspondingly when the surgeon encounters softer bone, the ability to drill accurately diminishes with the loss of tactile sensitivity and consequently inadvertent over-penetration and over-preparation of soft bone is common. Manufacturers make implants and components, but most of them do not actually treat patients. Their "scientific" assessments are usually based on marketing. We treat patients with implant components to their treatment plan every day and constantly evaluate our results, looking seriously at things that do not work and building on things that do. The end result is that we take a great number of patients who have significant dental problems and treat them successfully using a dental implant-based protocol. We constantly evaluate our results, looking seriously at things that do not work and building on things that do. Because we do so much of this, we know what actually works and what doesn't. Limiting implantology to drilling holes further deprives patients of that precious basic building block of implantology-the bone.

The Bone Renaissance technique offer a useful and predictable procedure, improved tactile sensitivity, improved control, and improved implant placement in maxillary \& mandibular atrophic ridges, crestal sinus floor elevations, post-extraction immediate implants and compromised soft bone conditions. It enhances the ability to manipulate and expand the alveolar walls and interradicular crests thereby resulting in an ideal increased number of sites that may be amenable to immediate post-extraction

implant placement and avoid the need for multiple-stage procedures.

\section{Clinical Technique}

The atraumatic, innovative, essentially a flapless surgical technique is designed to expand an initial osteotomy created by inserting the scalpel blade first by pushing and then by careful malleting to bisect attached gingiva and ridge. The socket development begins with the use of the blade \#15 to separate the labial and the lingual plates. Blade is tapped between the buccal and lingual walls followed by the instrument \#2 which is slightly more wider than the blade. Specially developed and designed bone osteotomes are used to expand narrow ridges to improve the density and complete molding of the bone against the sides of the implant site (Figure 4).

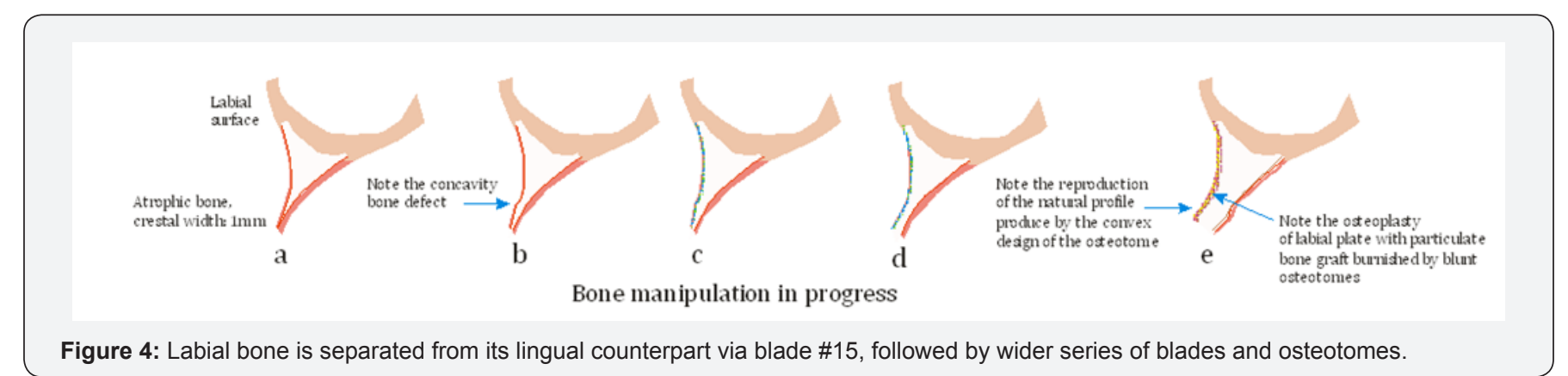

In extremely dense and atrophic mandibular ridges, the socket development begins with the use of the blade \#15 to separate the labial and the lingual plates, followed by the instrument \#2 open the crestal window to introduce the sharp pointed pilot drill of
$1.5 \mathrm{~mm}$ diameter at a speed of $1500-2000 \mathrm{rpm}$ with profused irrigation to a depth of $8 \mathrm{~mm}$ to $10 \mathrm{~mm}$, creating an osteotomy of $1.5 \mathrm{~mm}$ in diameter (Figure 5). 


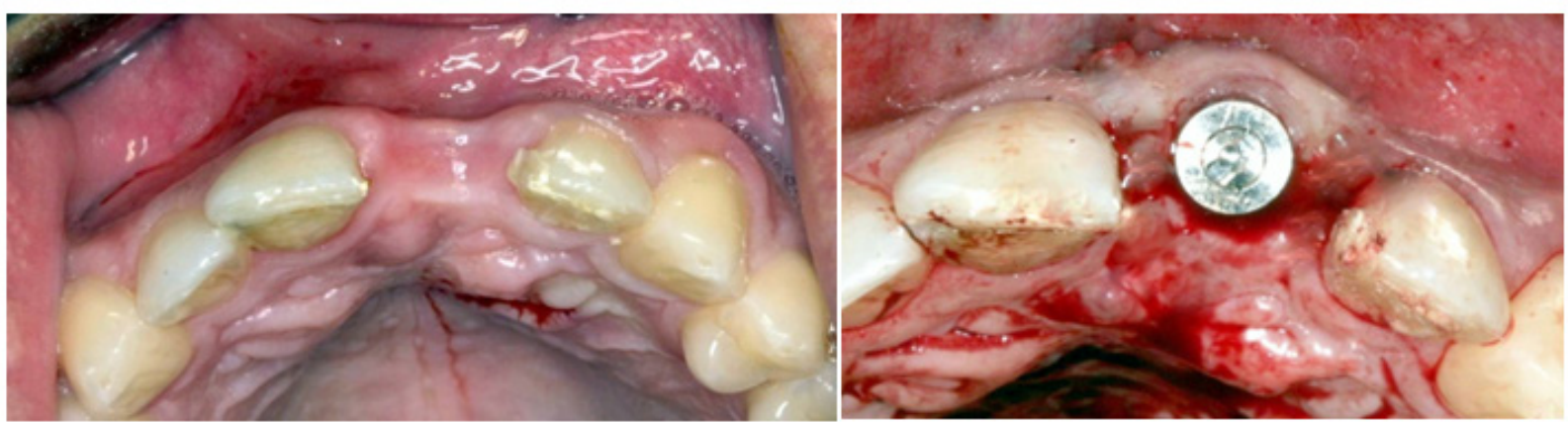

Figure 5: Note the reproduction of the natural facial bone contour over the implant picture.

As instruments are introduced into the bone, cortical and cancellous bone is enthused outwards compacting it against the face of implant socket. Use $1.5 \mathrm{~mm}$ in diameter straight manual osteotome for anteriors and offset for posteriors. Gently push and tap into the initial site, followed by $2 \mathrm{~mm}$ wider until you reach the desired depth. Next connect the motor driven osteotome \# 1 in the hand piece with a speed of no more than $50 \mathrm{rpm} \&$ torque between 15-25N followed by \# 2 \& 3 . The specific drill and bone expander sequence may vary according to the bone quality and quantity and the desired diameter of the implant. Drilling bone to prepare implant sites is only required in situations where extremely dense bone is encountered. In such situations widen the opening so that a drill may be inserted easily without touching the crestal bone. If this crestal bone is too thin less than 0.75 or so then do some augmentation by adding and burnishing particulate graft. If a resistance is encountered due to the cortical bone just take a $2 \mathrm{~mm}$ diameter drill and gently shave off the bone beside the hole. If the bone is relatively soft (as in maxilla) don't use the drills it would be more desirable to use a combination of hand or motor driven osteotomes according to convenience and approach. Expansion should be switched to a hand ratchet and the instruments may be inserted at intervals, slow motion pausing for a while to allow time for blood circulation and the bone to expand, introducing progressively larger osteotomes, as needed and repeating the rotating and manual strokes.

Continually enlarge the opening by utilizing a combination of motor-driven \& mallet-driven bone expanders together with an amalgamation of autologous growth factors in a sequential, codified approach to achieve an unsurpassed implant stability. The density of the bone adjoining the implant in so doing is tremendously improved and augmented. Osteoplasty is achieved by using a series of blunt osteotomes which are repeatedly moved forwards \& backwards to amalgamate and burnish the particulate bone in the osteotomy together with concentrated growth factors collected from the patient's autologous blood and this simultaneously augment and enlarge the osteotomy up to the desirable width. With this, the bone layer next to the osteotomy is improved because of the compaction of bone which in turn helps anchor a desirable endosseous implant (Figure 6).
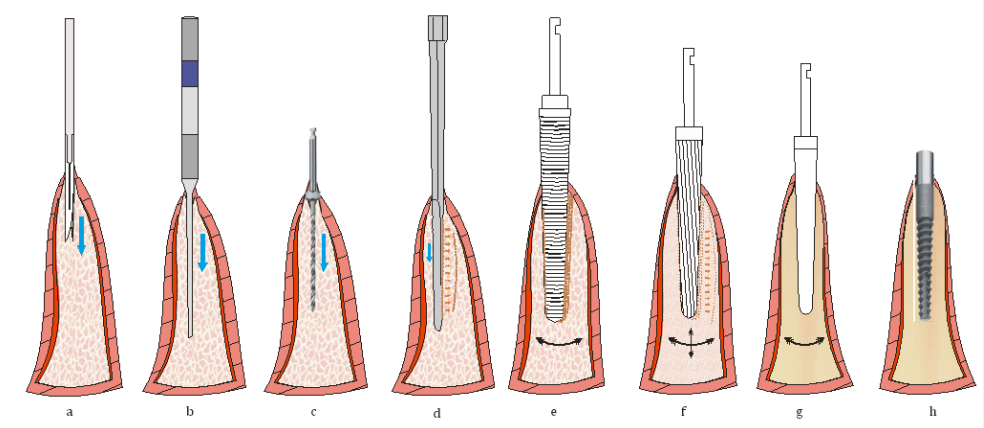

Figure 6: Stages of bone renaissance manipulation.

The Bone Renaissance may include a very few surgical burs (2-3) that helps allow drilling into the cortical bone to improve control of the expansion so that the implant may ultimately be placed in an appropriately centered position within the expanded crest.

Immediate Implant Socket Development: In cases of Immediate implants in multi-rooted teeth, the most desirable socket is selected and the bone manipulation is initiated at a more palatal position, and then mechanically oriented in the desired direction by applying pressure on the bone expanders/ osteotomes as it manipulates, rotates while advancing into the atrophic ridge along with the repositioning, directing, gently pushing, molding manually and mechanically together with the addition of particulate graft. The expanders are driven at 
speeds between $20 \mathrm{rpm}$ - $50 \mathrm{rpm}$ with torque about $15-25 \mathrm{Ncm}$ Rotary expanders may additionally be used to pack the grafting material. Although the narrower bone expanders may be utilized to penetrate the sinus floor, caution must be taken because of the increased risk of perforating the Schneiderian membrane. Blunt apical design, expanders are designed for this purpose. Use of Bone Renaissance concept to expand an osteotomy by burnishing helps to maintain all of the existing bone material by pushing the bone aside with minimal trauma while developing an accurately shaped osteotomy. Smooth-sided osteotomes are used to burnish the graft and further condense the bone, while the threaded design of the motor-driven bone expanders prepares taps in the osteotomy site where there is extremely dense bone that further facilitate the subsequent insertion of a threaded implant, and greatly enhances the initial stability as well (Figure 7).
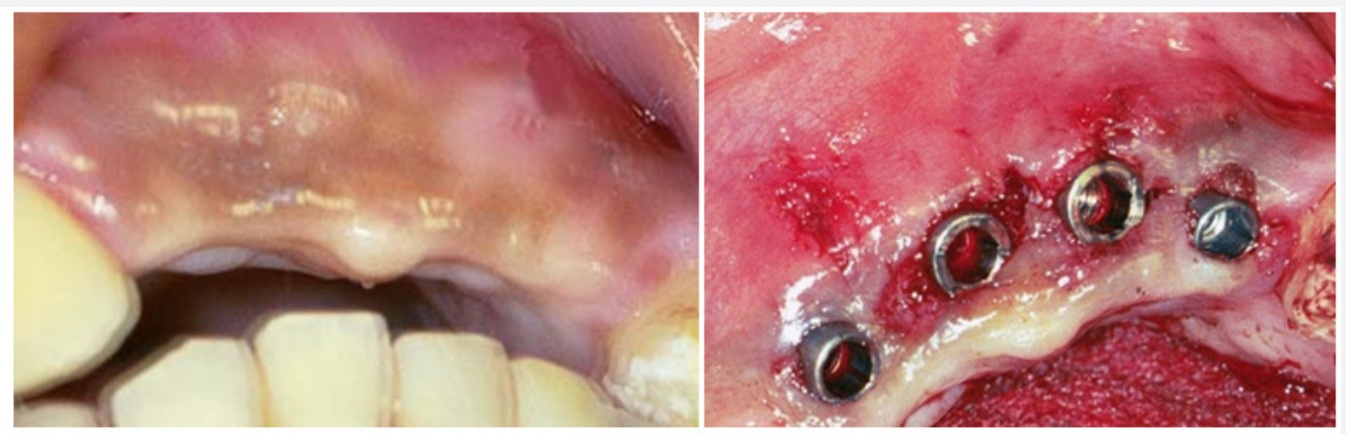

Figure 7: Bone renaissance makes it possible to treat such an advanced atrophic ridge, less than $1.5 \mathrm{~mm}$ so manipulated to receive the most appropriate standard implants.

The clinical experience suggests that Bone Renissance may provide increased control over the expansion site, therefore allowing treatment of more severely atrophic ridges than previously possible with traditional systems. In severely atrophic bone once the plastic capacity of the bone has been exceeded, this technique allows a gradual and controlled fracture of the buccal plate that may be deliberately induced to meet the expansion requirements. The displacement of the fractured segments may be closely scrutinized and, as long as adequate implant stability is achieved, the fracture site may be grafted and implant placement may be accomplished with a single-stage approach.

The clinical significance of this is that the crestal bone and tissue margins are much more stable in correctly manipulated bone than in grafted bone [7].

\section{Bone renissance treating the vertical deficiencies; treatment of inadequate ridge height}

Vascularized crestal bone can predictably and simply be repositioned to regain height with segmental osteotomies and later implants can be placed with bone expansion. In this bone renaissance component, remote relieving incisions are given followed by sectioning of the part of the ridge to be repositioned via a tunnel approach. The sectioned segment is moved into the desired position and thereby stabilized resulting in an immediate overbuilt with improved gingival contour, greater height for implant placement. Blood supply to the block is maintained throughout the single surgical procedure as periosteum remains attached to the section with immediate accurate repositioning. The transferred bone with attached periosteum is superior to traditional grafting as here there is more stable bone, natural implant position and profile, reduced risks of complications, shorter treatment time and significantly lesser costs.

\section{Bone renaissance soft tissue management}

Stable band of attached gingiva around every implant can be achieved at time of implant placement with tissue manipulation or tissue grafting.

\section{Bone renaissance treating the sinus deficiencies:}

(a)

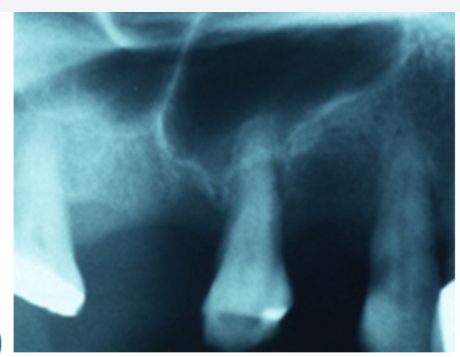

(b)

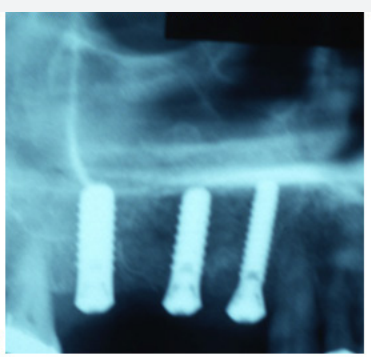

Figure 8: (a) Extreme pneumatization of the sinus with a $\mathrm{mm}$ of bone displayed at the sinus floor (b) Sinus grafted and replaced with $17 \mathrm{~mm}$ long implants 
Maxillary sinus expands inferiorly and laterally after loss of posterior teeth. It may progress to take up whole posterior maxilla, and is a pneumatized with 1 to $2 \mathrm{~mm}$ of bone below the sinus floor. Sinus lining is raised to sandwich graft material between sinus lining and sinus floor to gain bone height to place implants in the maxillary posterior region (Figure 8).

\section{Conclusion}

The use of bone renaissance concept has the potential of treating both thin \& vertically deficient ridges as long as the periosteum and soft tissues over the alveolar bone are respected. The bone keeps its inherent potential of expansion and can be moved vascularized to the desirable position to regain lost height.

The purpose of this presentation is to help develop an understanding that the benefits of the multi- modality approach to implant dentistry cannot be denied. The various vistas opened up by the particular applications and benefits of new techniques can dramatically increase the scope of treatment, thus enabling surgeons to provide the benefits to a greater number of patients with long term predictable and successful results to restore the patients back to ful

\section{References}

1. Tatum OH, Lebowitz MS (1991) Anatomic considerations for dental implants. J Oral Implantology17(1): 16-21.

2. Misch CE (1988) Contemporary Implant Dentistry. ( $3^{\text {rd }}$ edn), Elsevier, USA.

3. Roberts WE, Turley PK, Brezniak N, Fielder PJ (1987) Implants: Bone physiology and metabolism. CDA J 15(10): 54-61.

4. Summers RB (1994) A new concept in maxillary implant surgery: The osteotome technique. Compendium 152(2): 154-156.

5. Januário AL, Duarte WR, Barriviera M, Mesti JC, Araújo MG, et al. (2011) Dimension of the facial bone wall in the anterior maxilla: a cone-beam computed tomography study. Clin Oral Implants Res 22(10): 11681171.

6. Chanavaz M (1988) Anatomy and histophysiology of the periosteum: quantification of the periosteal blood supply to the adjacent bone with 85Sr and gamma spectrometry. J Oral Implantol 21(3): 214-219.

7. Tatum H (1986) Maxillary and snus implant reconstructions. Dent Clin North Am 30(2): 207-229.

\section{Your next submission with Juniper Publishers} will reach you the below assets

- Quality Editorial service

- Swift Peer Review

- Reprints availability

- E-prints Service

- Manuscript Podcast for convenient understanding

- Global attainment for your research

- Manuscript accessibility in different formats

(Pdf, E-pub, Full Text, Audio)

- Unceasing customer service

Track the below URL for one-step submission https://juniperpublishers.com/online-submission.php 